Cite this article as: BMJ, doi:10.1136/bmj.39196.740995.BE (published 21 May 2007)

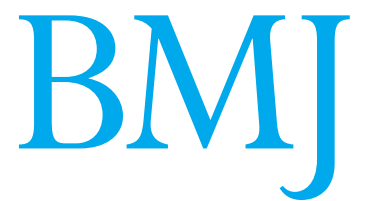

RESEARCH

\title{
Accuracy of liquid based versus conventional cytology: overall results of new technologies for cervical cancer screening randomised controlled trial
}

\author{
Guglielmo Ronco, responsible, Cervical Screening Evaluation Unit , ${ }^{1}$ Jack Cuzick, director , ${ }^{12}$ Paola \\ Pierotti, senior biologist, ${ }^{8}$ Maria Paola Cariaggi, director, Unit of Cytopathology, ${ }^{3}$ Paolo Dalla Palma, director, \\ Unit of Pathology, ${ }^{4}$ Carlo Naldoni, coordinator regional cancer screening programmes, ${ }^{7}$ Bruno \\ Ghiringhello, director, Unit of Pathology, ${ }^{2}$ Paolo Giorgi-Rossi, responsible, ${ }^{10}$ Daria Minucci, director , ${ }^{6}$ Franca \\ Parisio, senior biologist, ${ }^{2}$ Ada Pojer, senior biologist, ${ }^{4}$ Maria Luisa Schiboni, senior biologist, ${ }^{11}$ Catia \\ Sintoni, senior biologist, ${ }^{9}$ Manuel Zorzi, epidemiologist, ${ }^{5}$ Nereo Segnan, director, ${ }^{1}$ Massimo \\ Confortini, director, Unit of Analytic and Biomolecular Cytology ${ }^{3}$, and the New Technologies for Cervical \\ Cancer Screening (NTCC) Working Group
}

\section{${ }^{1}$ Unit of Cancer Epidemiology, \\ Centre for Cancer Prevention, \\ Via S Francesco da Paola 3110123 \\ Turin, Italy \\ ${ }^{2} \mathrm{~S}$ Anna Hospital, Turin, Italy \\ ${ }^{3}$ Scientific Institute for Cancer \\ Prevention of Tuscany Region, \\ Florence, Italy \\ ${ }^{4} \mathrm{~S}$ Chiara Hospital, Trento, Italy \\ ${ }^{5}$ Venetian Tumour Registry, \\ Istituto Oncologico Veneto, \\ Padua, Italy}

${ }^{6}$ Unit of Obstetrics and

Gynaecology, University Hospital,

Padua, Italy

${ }^{7}$ Emilia-Romagna Region,

Bologna, Italy

${ }^{8}$ Maggiore Hospital,

AUSL Bologna, Italy

${ }^{9} \mathrm{~S}$ Maria delle Croci Hospital,

Ravenna, Italy

${ }^{10}$ Health Technologies Unit,

Agency for Public Health

Lazio Region, Rome, Italy

${ }^{11} \mathrm{~S}$ Giovanni Hospital, Rome, Italy

${ }^{12}$ Cancer Research UK Clinical Centre, Epidemiology,

Mathematics and Statistics,

Queen Mary's School of Medicine and Dentistry, London

Correspondence to: $\mathrm{G}$ Ronco

guglielmo.ronco@cpo.it

doi: 10.1136/bmj.39196.740995.BE

\section{ABSTRACT}

Objective To compare the accuracy of conventional cytology with liquid based cytology for primary screening of cervical cancer.

Design Randomised controlled trial.

Setting Nine screening programmes in Italy.

Participants Women aged 25-60 attending for a new

screening round: 22466 were assigned to the

conventional arm and 22708 were assigned to the

experimental arm.

Interventions Conventional cytology compared with

liquid based cytology and testing for human

papillomavirus.

Main outcome measure Relative sensitivity for cervical intraepithelial neoplasia of grade 2 or more at blindly reviewed histology, with atypical cells of undetermined significance or more severe cytology considered a positive result.

Results In an intention to screen analysis liquid based cytology showed no significant increase in sensitivity for cervical intraepithelial neoplasia of grade 2 or more (relative sensitivity $1.17,95 \%$ confidence interval 0.87 to 1.56) whereas the positive predictive value was reduced (relative positive predictive value $v$ conventional cytology $0.58,0.44$ to 0.77 ). Liquid based cytology detected more lesions of grade 1 or more (relative sensitivity $1.68,1.40$ to 2.02), with a larger increase among women aged 25-34 ( $P$ for heterogeneity 0.0006), but did not detect more lesions of grade 3 or more (relative sensitivity $0.84,0.56$ to 1.25$)$. Results were similar when only low grade intraepithelial lesions or more severe cytology were considered a positive result. No evidence was found of heterogeneity between centres or of improvement with increasing time from start of the study. The relative frequency of women with at least one unsatisfactory result was lower with liquid based cytology $(0.62,0.56$ to 0.69).
Conclusion Liquid based cytology showed no statistically significant difference in sensitivity to conventional cytology for detection of cervical intraepithelial neoplasia of grade 2 or more. More positive results were found, however, leading to a lower positive predictive value. A large reduction in unsatisfactory smears was evident. Trial registration Current Controlled Trials ISRCTN81678807.

\section{INTRODUCTION}

Liquid based cytology is used widely for primary screening of cervical cancer but high quality studies on its accuracy are limited. Indeed almost all published studies are based either on the comparison of non-randomly assigned populations or on double testing the same women. With the double testing design, cells used for diagnosis could be removed with the conventional sample, which is taken first, leading to an underestimation of the accuracy of liquid based cytology. In addition only some of the published studies, in a primary screening setting, considered histologically confirmed cervical intraepithelial neoplasia as the end point and only a few verified the diagnosis by colpohistology in the large majority of women with abnormal cytology results. A recent review identified one small randomised trial only and concluded that large randomised trials were needed. ${ }^{1}$

We carried out a large randomised trial, over two phases, on new technologies for cervical cancer screening, including liquid based cytology and testing for human papillomavirus. The main final end point of the study will be long term rates of disease. Results at recruitment from phase 1-mainly focused on testing for human papillomavirus - have been published for women aged $35-60^{2}$ and $25-34 .^{3}$ We compared conventional cytology with liquid based cytology for the entire age range (25-60). We also studied the influence 
of women's age and interpreter's experience on the accuracy of liquid based cytology.

\section{METHODS}

We carried out a randomised controlled trial in nine cervical cancer screening centres in Italy. These centres routinely invite women aged 25-64 for a smear test every three years. Methods of recruitment and randomisation have been described. ${ }^{23}$ Briefly, during 2002 3 , after written informed consent had been obtained, women aged 25-60 attending for a new routine screening round were alternately randomised by smear takers to conventional cytology or to liquid based cytology and testing for human papillomavirus. Randomisation was generated by computer (two centres, unblocked) or by the opening of sequentially numbered sealed envelopes (blocks of eight in three centres, unblocked in four). Women who consented to receive the results were then told the outcome. We excluded women who were pregnant, had undergone hysterectomy, or had been treated for cervical intraepithelial neoplasia within five years.

A sample of cervical cells was taken using a plastic Ayre's spatula and cytobrush. A standard glass slide was prepared for women randomised to the conventional arm. For women randomised to the experimental arm cells were put into PreservCyt solution (ThinPrep; Cytyc, Boxborough, MA) and used to make liquid based cytology preparations and to test for human papillomavirus. The slides were read in 14 laboratories participating in regular screening programmes, without knowledge of the results for human papillomavirus. The same cytologists read both the liquid based and the conventional slides in each centre. Slides showing abnormal results were reviewed by a supervisor (or by a panel of cytologists in Florence) before the women were given the results. The diagnosis was used for the women's management and study analysis.

Cytology was classified according to the Bethesda 1991 system except that we did not use the subcategories for atypical cells of undetermined significance. Two laboratories had no experience of interpreting liquid based slides whereas seven had experience with ThinPrep (range 500 to 10000 slides read per laboratory) and five had experience with another liquid based system (about 1000 slides read per laboratory). The laboratories routinely carried out quality assurance of cytology, including monitoring the distribution of diagnoses and positive predictive values and the circulation and discussion of the results of smear tests within and between laboratories. Quality assurance continued during the study period. To improve consistency between centres, 30 liquid based slides that were considered difficult to classify were circulated during the study and blindly read by each participating centre. Slides with discrepant results were discussed between representatives of each centre.

Women in the experimental arm were referred to colposcopy if cytology showed atypical cells of undetermined significance or more severe results. Women with normal cytology but positive for human papillomavirus were referred directly to colposcopy if they were aged 35-60 but if aged 25-34 were recalled for testing after one year. These results are not considered in the present analysis. Women in the conventional arm were managed according to the standard protocol of each centre. They were referred for colposcopy if cytology showed a low grade or higher squamous intraepithelial lesion. In seven centres ( $72 \%$ of randomised women), women with atypical cells of undetermined significance were referred directly to colposcopy whereas the remaining two centres recommended repeat testing and referral for colposcopy if the result was at least low grade intraepithelial lesions.

In each centre the same colposcopists examined women in both arms. They had access to the patients' cytology and human papillomavirus results. Biopsy samples were taken from suspicious areas.

Our primary end point was histologically confirmed cervical intraepithelial neoplasia of grade 2 or more detected during the recruitment phase as a result of abnormal cytology. Therefore for the present analyses we did not consider the results of cytological and histological tests that were carried out because of a positive human papillomavirus test result in the presence of normal cytology. These tests would not have been carried out if cytology alone was used. Operationally we included lesions detected within one year from referral to colposcopy. The biopsy results for women with a histologically confirmed cervical intraepithelial neoplasia were reviewed independently and blindly to study arm and cytology result. ${ }^{2}$

\section{Statistical analysis}

We calculated the relative frequency of cytology results, including unsatisfactory results in the experimental arm compared with the conventional arm.

Table 1 | Proportion of women with unsatisfactory cytology results by cytology group. Values are percentages (numbers) unless stated otherwise

\begin{tabular}{|c|c|c|c|}
\hline Unsatisfactory cytology* & Conventional group $(n=22466)$ & Liquid based group $(n=22708)$ & $\begin{array}{l}\text { Relative frequency } \dagger \\
(95 \% \mathrm{Cl})\end{array}$ \\
\hline Any reason & $4.11(923)$ & $2.57(583)$ & $0.62(0.56$ to 0.69$)$ \\
\hline Obscuring inflammation & $2.15(483)$ & $0.44(100)$ & $0.21(0.17$ to 0.25$)$ \\
\hline Other & $1.96(440)$ & $2.13(483)$ & 1.09 (0.96 to 1.23$)$ \\
\hline
\end{tabular}

*Women with at least one unsatisfactory result.

tRatio of percentages. Liquid based cytology compared with conventional cytology. 
Table 2 | Most severe cytology finding in screening episode, by cytology group. Values are percentages (numbers) unless stated otherwise

\begin{tabular}{|c|c|c|c|}
\hline Finding & Conventional group $(n=22466)$ & Liquid based group $(n=22708)$ & Relative frequency* $(95 \% \mathrm{Cl})$ \\
\hline ASCUS/AGUS & $2.29(514)$ & $3.59(815)$ & 1.57 (1.41 to 1.75$)$ \\
\hline \multicolumn{4}{|c|}{ Intraepithelial lesions: } \\
\hline High grade & $0.26(58)$ & $0.41(92)$ & 1.57 (1.13 to 2.18 ) \\
\hline
\end{tabular}

ASCUS=atypical squamous cells of undetermined significance; AGUS=atypical glandular cells of undetermined significance.

*Ratio of percentages. Liquid based cytology compared with conventional cytology.

Given the randomised design, the same number of lesions was expected in each of the arms except for random variation and differences in the actual detection attributed to differences in sensitivity. Therefore we estimated the relative sensitivity of liquid based cytology compared with conventional cytology as the relative detection rate compared with the conventional arm. We included all randomised eligible women in an intention to screen analysis. The relative positive predictive value of liquid based cytology compared with conventional cytology was calculated only for women who underwent colposcopy. We calculated confidence intervals using methods appropriate for ratios of independent proportions. We also calculated relative sensitivity and positive predictive value when considering only low grade intraepithelial lesions or more severe cytology as a positive result (cut-off point was low grade intraepithelial lesions).

We considered potential effect modifiers of relative sensitivity and relative positive predictive value: age (25-34 v 35-60), centre, protocol for dealing with women with atypical cells of undetermined significance in the conventional arm (centres directly referring women to colposcopy $v$ those advising repeat testing), and experience with liquid based cytology (centres with experience of ThinPrep, of other types of liquid based cytology, or no experience with liquid based cytology). We tested for heterogeneity across different groups using the Breslow-Day test. In addition we studied the learning effect by considering the women's date of recruitment as a modifier with linear effect, using unconditional logistic regression and adjusting by centre. SAS software version 8.2 was used for analyses. $\mathrm{P}$ values are two sided.

The main final end point of the entire study will be the long term rates of disease. The size (about 100000 women) of the entire two phase study (present study and study testing for human papillomavirus only in experimental arm) was determined for $80 \%$ power to show a significant reduction (relative detection rate 0.68 ) in the detection rate for cervical intraepithelial neoplasia of grade 2 or more in the experimental arm compared with the conventional arm at the screening round after recruitment. ${ }^{2}$ On the other hand, at recruitment we anticipated an increase in sensitivity in the experimental arm. With the obtained study size in the first phase of the study and with the observed detection rate of cervical intraepithelial neoplasia of grade 2 or more of $0.37 \%$ in the conventional arm, the study had over $80 \%$ power to detect as significant a $50 \%$ increase in sensitivity (relative detection rate 1.5 ) with a two sided 5\% type I error probability and a $41 \%$ power for a $30 \%$ increase.

\section{RESULTS}

Overall, 22466 women were randomised to conventional cytology and 22708 to liquid based cytology for primary screening of cervical cancer. The median age was 41 in both arms ( $\mathrm{P}=0.34$ by median score test). In total, 49\% of women in both arms (10906 and $11149)$ had a cervical smear test registered in a programme within the past four years $(\mathrm{P}=0.24)$.

The figure shows the trial profile. Three hundred and one women in the experimental arm (1.3\%) had conventional cytology. At least one colposcopy was carried out in 93\% (1998/2154 of women referred because of abnormal cytology results: $91 \%$ (661/724) in the conventional arm and 93\% (1337/1430) in the experimental arm). Among women attending for colposcopy the mean number of colposcopies and mean number of biopsies in the conventional arm were 1.33 (SD 0.53) and $0.76(0.90)$ and in the experimental arm were $1.33(0.52)$ and $0.74(0.94)$.

The overall proportion of women with at least one unsatisfactory cytology result was significantly reduced with liquid based cytology (table 1). This reduction was larger $(\mathrm{P}=0.029)$ in women aged 25 -34 (relative frequency $v$ conventional cytology $0.53,95 \%$ confidence interval 0.44 to 0.63 ) than in women aged $35-60(0.67,0.59$ to 0.76$)$. The reduction was large for results considered unsatisfactory because of obscuring inflammation but not for other reasons (table 1).

The proportion of women with atypical squamous cells of undetermined significance or atypical glandular cells of undetermined significance and low and high grade squamous intraepithelial lesions was significantly increased in the experimental arm (table 2). The increase in atypical squamous or atypical glandular cells of undetermined significance was larger $(\mathrm{P}=0.0199)$ in women aged 25-34 (relative frequency $v$ conventional cytology $1.92,1.56$ to 2.36 ) than in women aged $35-60$ (1.44, 1.27 to 1.64$)$.

No significant increase was observed in sensitivity for cervical intraepithelial neoplasia of grade 2 or more for liquid based cytology compared with conventional cytology with either atypical cells of undetermined significance or low grade intraepithelial lesions as cut-off points (table 3). The positive predictive value, 
however, was significantly reduced for liquid based cytology when using any end point or cut-off point (table 3). When the analysis was restricted to centres referring all women with atypical cells of undetermined significance directly to colposcopy, using such cells as the cut-off point and cervical intraepithelial neoplasia of grade 2 or more as the end point the relative sensitivity was 1.11 and the relative positive predictive value was 0.65 .

Liquid based cytology showed an increased sensitivity for cervical intraepithelial neoplasia of grade 1 or more when atypical cells of undetermined significance or more severe cytology were considered and when low grade intraepithelial lesions were the cut-off point (table 3). This increased sensitivity was larger in younger women $(\mathrm{P}=0.0006$ with atypical cells as cutoff point and $\mathrm{P}=0.02$ with low grade lesions as cut-off point): with atypical cells as the cut-off point the relative sensitivity was 2.21 (95\% confidence interval 1.67 to 2.91 ) in women aged $25-34$ and 1.33 (1.04 to 1.70) in women aged 35-60. The corresponding values with low grade lesions as the cut-off point were 2.23 (1.60 to 3.11 ) and 1.32 (0.98 to 1.78 ). No increased sensitivity was observed with cervical intraepithelial neoplasia of grade 3 or more as the end point.

No significant heterogeneity was observed between centres for relative sensitivity (smallest $\mathrm{P}=0.58$ ) or relative positive predictive value (smallest $\mathrm{P}=0.13$ ) when cervical intraepithelial neoplasia of grades 2 or more or 3 or more were used as end points. When centres were compared according to experience with liquid based cytology significant variability was not found for relative sensitivity or for relative positive predictive values (smallest $\mathrm{P}=0.21$ ). In addition, after adjusting for centre no significant effect was observed for the interval from the start of the study either on relative sensitivity (smallest $\mathrm{P}=0.27$ ) or relative positive predictive value (smallest $\mathrm{P}=0.26$ ) with any end point or cut-off point. When the analysis was restricted to laboratories with experience of ThinPrep, the relative sensitivity and relative positive predictive value with atypical cells of undetermined significance as the cut-off point and cervical intraepithelial neoplasia of grade 2 or more as the end point were 1.12 and 0.63 , respectively. When only the second half of phase 1 of enrolment in each centre was considered the corresponding values were 1.04 and 0.54 .

\section{DISCUSSION}

Liquid based cytology for primary screening of cervical cancer showed a significantly increased sensitivity for cervical intraepithelial neoplasia of grade 1 but not for lesions of grade 3 or more. We observed a relevant reduction in unsatisfactory slides with liquid based cytology as a result of a decrease in obscuring inflammation. We observed a slight increase of the point estimate for relative sensitivity of liquid based cytology compared with conventional cytology for cervical intraepithelial neoplasia of grade 2 or more $(1.17$ with atypical cells of undetermined significance as the cutoff point, 1.03 with low grade intraepithelial lesions as the cut-off point) but this was far from significant.

Table 3 Relative sensitivity and relative positive predictive value of experimental (mainly liquid based cytology) compared with conventional arm (conventional cytology) for different histologically confirmed end points and with different cut-off points according to cytology result. Values are percentages (numbers) of women unless stated otherwise

Histological cervical intraepithelial neoplasia end point

Grade 1 or more Grade 2 or more Grade 3 or more

Positive if cytology shows atypical cells of undetermined significance or more

Detection rate:

\begin{tabular}{|c|c|c|c|}
\hline Conventional group & $0.82(184)$ & $0.37(84)$ & $0.24(53)$ \\
\hline Liquid based group* & $1.38(313)$ & $0.44(99)$ & $0.20(45)$ \\
\hline Relative sensitivity† ( $95 \% \mathrm{Cl})$ & 1.68 (1.40 to 2.02$)$ & $1.17(0.87$ to 1.56$)$ & $0.84(0.56$ to 1.25$)$ \\
\hline \multicolumn{4}{|l|}{ Positive predictive value: } \\
\hline Conventional group & 27.84 & 12.7 & 8.02 \\
\hline Liquid based group* & 23.41 & 7.4 & 3.37 \\
\hline Relative positive predictive valuet $(95 \% \mathrm{Cl})$ & $0.84(0.72$ to 0.98$)$ & $0.58(0.44$ to 0.77$)$ & $0.42(0.29$ to 0.62$)$ \\
\hline \multicolumn{4}{|c|}{ Positive if cytology shows low grade intraepithelial lesions or more } \\
\hline \multicolumn{4}{|l|}{ Detection rate: } \\
\hline Conventional group & $0.55(123)$ & $0.31(70)$ & $0.20(44)$ \\
\hline Liquid based group* & $0.95(211)$ & $0.32(73)$ & $0.14(32)$ \\
\hline Relative sensitivity† ( $95 \% \mathrm{Cl})$ & 1.70 (1.36 to 2.12$)$ & $1.03(0.74$ to 1.43$)$ & $0.72(0.46$ to 1.13$)$ \\
\hline \multicolumn{4}{|l|}{ Positive predictive value: } \\
\hline Conventional group & 38.80 & 22.08 & 13.88 \\
\hline Liquid based group* & 36.76 & 12.72 & 5.57 \\
\hline Relative positive predictive value† $(95 \% \mathrm{Cl})$ & $0.95(0.80$ to 1.13$)$ & $0.58(0.43$ to 0.78$)$ & $0.40(0.26$ to 0.62$)$ \\
\hline
\end{tabular}

For detection rate and relative sensitivity denominators are 22466 women in conventional group and 22708 in liquid based group.

For positive predictive values and relative positive predictive values denominators are women with positive cytology for atypical cells of undetermined significance who had had colposcopy: 661 in conventional group and 1337 in liquid based group.

* Only cases of cervical intraepithelial neoplasia detected by cytology considered.

tRatio of percentages. Liquid based cytology compared with conventional cytology. 


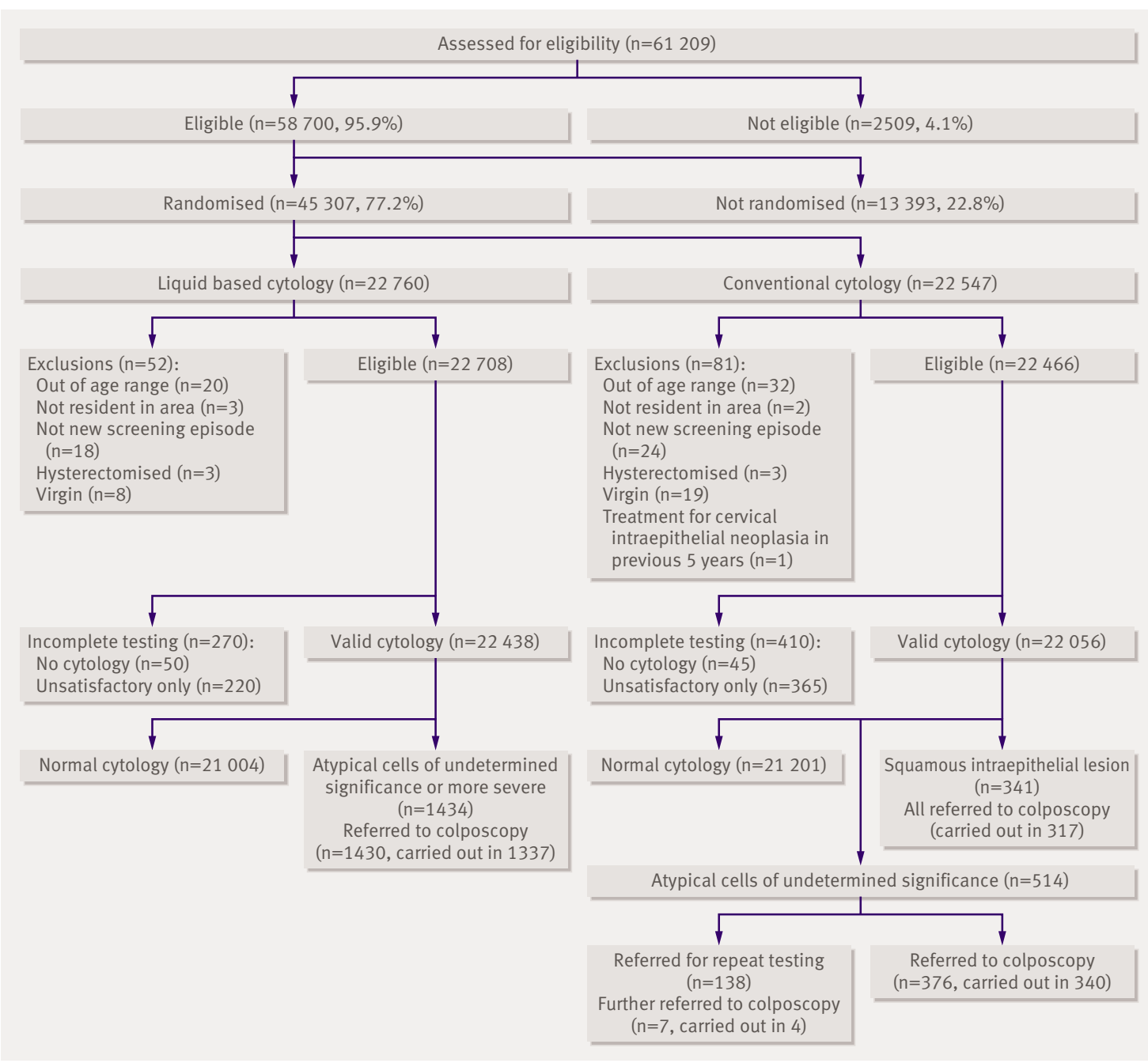

Trial profile

Values between 0.87 and 1.56 were included in the $95 \%$ confidence interval of relative sensitivity with atypical cells of undetermined significance as the cut-off point. Therefore we cannot exclude increases and decreases of sensitivity in this range. The study had a $80 \%$ power to detect as statistically significant a $50 \%$ increase in sensitivity and a $41 \%$ power to detect a $30 \%$ increase. The study size was, however, determined for another objective (long term rates of cervical intraepithelial neoplasia). These power calculations are based on the observed number of women recruited and the detection rate in the liquid based cytology arm.

We carried out a large randomised trial nested in screening programmes, which was representative of routine activity. Colposcopy was carried out in a high proportion of women whose screening results were positive. Colposcopists were not blinded to type of cytology, but the number of biopsies per woman undergoing colposcopy was similar in the two arms. Histology was independently reviewed, with reviewers blinded to trial arm and cytology result. A few centres adopted a different protocol between study arms for the management of women with atypical cells of undetermined significance. However the results were almost unchanged when the analysis was restricted to the centres that applied the same protocol in both arms and when low grade intraepithelial lesions were considered as the cut-off point for cytology.

Several reviews and meta-analyses of the performance of liquid based cytology, based on non-randomised studies, have been published but have reached conflicting conclusions. ${ }^{1-9}$ A recent systematic review on liquid based cytology ${ }^{1}$ found one small randomised controlled trial only. ${ }^{10}$ The same review ${ }^{1}$ identified only five "high quality" studies (slides read without knowledge of the others' results, with verification by masked reference standard of at least all positive slide results). ${ }^{11-15}$ Such studies did not show increased accuracy with liquid based cytology, in agreement with our results. However four of them were split sampled and this could affect the accuracy of liquid based cytology.

We also found that liquid based cytology had a lower positive predictive value than conventional cytology. This reduction was the result of an increased frequency 


\section{WHAT IS ALREADY KNOWN ON THIS TOPIC}

Despite the widespread use of liquid based cytology only a few high quality studies and no large randomised trial have examined its diagnostic accuracy

Systematic reviews have produced conflicting conclusions

\section{WHAT THIS STUDY ADDS}

Liquid based cytology shows no significant difference in sensitivity to conventional cytology A significant reduction in positive predictive value was, however, apparent

of abnormal findings (usually low grade) without an increase in high grade cervical intraepithelial neoplasia on histology. An increased frequency of low grade lesions with liquid based cytology has already been observed. ${ }^{1}$ The relative frequency of atypical cells of undetermined significance varied between studies, but overall in high and medium quality studies more slides were classified as atypical cells by liquid based cytology than by conventional cytology. ${ }^{1}$

Interpretation of cytology is highly subjective. Therefore the effect of moving from conventional to liquid based cytology could vary across laboratories. Our data do not, however, show evidence of heterogeneity between the centres in this study. We also did not observe any difference in the performance of liquid based cytology compared with conventional cytology according to previous experience with liquid based cytology, nor with increasing experience in the study. Although the power of interaction test is usually limited, in this case all $P$ values were far from significant. In addition, point estimates changed little when we restricted the analysis to centres with experience of ThinPrep, or to the second half of enrolment in each centre. It is possible that greater experience could have an effect but, in case of shift from conventional to liquid based cytology, this would mean a long period of decreased accuracy before possible advantages.

On the basis of this analysis, the main advantage of moving to liquid based cytology is a reduction in the rate of unsatisfactory slides. Other established advantages are the shorter time needed for interpretation ${ }^{1216}$ and the possibility of using the same sample for testing for human papillomavirus and for other molecular tests.

We thank the staff who helped with the study and the women who participated in this study.

Contributors: GR was the project leader and is guarantor. GR designed the study with NS and JC. GR, MPC, and JC drafted the manuscript. GR, PG-R, and MPC were responsible for data analysis. PDP, MPC, DM, FP, AP, MLS, and CS were locally responsible for preparation and interpretation of slides produced after liquid based cytology. MC and BG supervised the quality assurance for cytology interpretation. PDP and BG coordinated the review of histology. MC, $\mathrm{PDP}, \mathrm{PG}-\mathrm{R}, \mathrm{CN}$, and $\mathrm{MZ}$ organised the local fieldwork. All authors critically revised the manuscript. In addition to the authors the following are contributing members of the New Technologies for Cervical Cancer Screening (NTCC) Working Group_-Turin: R Rizzolo, D Mari, P Giubilato (CPO Piemonte), A Gillio-Tos, L De Marco (Unit of Cancer Epidemiology and CPO, CERMS, University of Turin), R Volante (Centre for early cancer diagnosis and treatment, OIRM S Anna), E Berardengo (Unit of Pathology, Ospedale S Giovanni AS), A Andrion (Unit of Pathology, Ospedale Martini), S Coverlizza (Unit of Pathology, Ospedale Giovanni Bosco), S Taraglio (Unit of Pathology, Ospedale Maria Vittoria), and MG Accinelli (Unit of Pathology, University of Turin). Trento:
E Polla, S Girlando, and D Aldovini (Unit of Pathology, Ospedale di Trento); Veneto: M Vettorazzi (Venetian Tumour Registry, Istituto Oncologico Veneto), A Del Mistro (Istituto Oncologico Veneto, Padua), M Matteucci (Unity of Gynaecology, Azienda Ospedaliera di Padova), L Onnis, E Insacco (Department of Pathology, University of Padua), M Lestani (Department of Pathology, University of Verona), and A Vignato (Servizio di Citologia, Ostetricia e Ginecologia, Ospedale di Soave); Emilia-Romagna: M Manfredi (Centro screening, AUSL Bologna), GP Casadei (Unit of Pathology, Ospedale Maggiore, AUSL Bologna), G Collina (Unit of Pathology Ospedale Bellaria, AUSL Bologna), M Serafini, P Schincaglia, B Vitali (Centro Prevenzione Oncologica, AUSL Ravenna), M Aldi (Unit of Pathology, Presidio Ospedaliero di Faenza, AUSL Ravenna), and S Folicaldi, R Nannini, G Galanti, and M De Lillo (Unit of Pathology, Presidio Ospedaliero, AUSL di Imola); Florence: M Zappa, A lossa, S Ciatto, S Cecchini, C Sani, F Carozzi (CSPO Firenze), and GL Taddei (Unit of Pathology University of Florence); Lazio: S Brezzi, P Raggi, and E Gomes (Local Health Unit, Viterbo, Italy), and A Pellegrini (Ospedale S Giovanni, Rome).

Funding: European Union (Europe against cancer contracts SI.2.327046 and SPC.2002475), Italian Ministry of Health (applied research projects and L 138/ 2004), Compagnia di S Paolo FIRMS, Regione Piemonte, Regione Toscana, Regione Veneto, Regione Emilia-Romagna, Agenzia di Sanità Pubblica, Regione Lazio. Funders had no role in study design, data collection, data analysis, data interpretation, or writing of the report

Competing interests: JC is a member of the speaker's bureau for Digene. His institution (Cancer Research UK) has received research funding from Roche

Diagnostics for a different study. MC is the principal recipient of a grant to the Scientific Institute for Cancer Prevention of Tuscany Region from Menarini Diagnostics. This grant is for another study.

Ethical approval: This study was approved by the local research ethics committees of the participating centres.

1 Davey E, Barrat A, Irwig L, Chan SF, Macaskill P, Mannes P, et al. Effect of study design and quality on unsatisfactory rates, cytological classification, and accuracy in liquid-based versus conventional cervical cytology: a systematic review. Lancet 2006;367:122-32.

2 Ronco G, Segnan N, Giorgi-Rossi P, Zappa M, Casadei GP, Carozzi F, et al. Human papillomavirus testing and liquid-based cytology: results at recruitment from the New Technologies for Cervical Cancer randomized controlled trial. / Natl Cancer Inst 2006:98:765-74.

3 Ronco G, Giorgi-Rossi P, Carozzi F, Dalla Palma P, Del Mistro A, De Marco L, et al. Human papillomavirus testing and liquid-based cytology in primary screening of women younger than 35 years: results at recruitment for a randomised controlled trial. Lancet Oncol 2006;7:547-55

4 Nanda K, McCrory DC, Myers ER, Bastina LA, Hasselblad V, Hickey JD, et al. Accuracy of the Papanicolau test in screening and follow-up of cervical cytologic abnormalities: a systematic review. Ann Intern Med 2000;132:810-9.

5 Bernstein SJ, Sanchez-Ramos L, Ndubisi B. Liquid-based cervical cytologic smear study and conventional Papanicolau smears: a meta analysis of prospective studies comparing cytologic diagnosis and sample adequacy. Am J Obstet Gynecol 2001;185:308-17.

6 Sulik SM, Kroeger K, Schultz JK, Brown JL, Becker LA, Grant WD. Are fluid-based cytologies superior to the conventional Papanicolau test? A systematic review. J Fam Pract 2001;50:1040-6.

7 Moseley RP, Paget S. Liquid-based cytology: is the way forward? Cytopathology 2002;13:71-82.

8 Abulafia O, Pezzullo JC, Sherer DM. Performance of ThinPrep liquid based cervical cytology in comparison with conventionally prepared Papanicolau smears: a quantitative survey. Gynecol Oncol 2003;90:137-44.

9 Klinkhamer PJ, Meerding WJ, Rosier PF. Hanselaar AG. Liquid-based cervical cytology Cancer 2003;99:263-71.

10 Obwegeser $\mathrm{H}$, Brack S. Does liquid-based technology really improve detection of cervical neoplasia? A prospective, randomized trial comparing the ThinPrep Pap Test with the conventional Pap Test, including follow-up of HSIL cases. Acta Cytol 2001;45:709-14.

11 Ferenczy A, Robitaille J, Franco E, Arseneau J, Richart RM, Wright TC. Conventional cervical cytologic smears: a paired comparison study on cervical cytology. Acta Cytol 1996;40:1136-42.

12 Sherman ME, Mendoza M, Lee KR, Ashfaq R, Birdsong GG, Corkill ME, et al. Performance of liquid-based, thin layer cervical cytology: correlation with reference diagnoses and human papillomavirus testing. Mod Pathol 1998;11:837-43.

13 Bergeron C, Bishop J, Lemarie A, Cas F, Ayivi J, Huynh B, et al. Accuracy of thin-layer cytology in patients undergoing cervical cone biopsy. Acta Cytol 2001;45:519-24.

14 Hessling JJ, Raso DS, Schiffer B, Calicott J, Husain M, Taylor D. Effectiveness of thin-layer preparation vs. conventional Pap smears in a blinded split-sample study. Extended cytologic evaluation. J Reprod Med 2001;46:880-6. 
15 Coste J, Cochand-Priollet B, De Cremoux P, Le Glès C, Cartier I, Molinié $V$, et al. Cross sectional study of conventional cenvical smear, monolayer cytology, and human papillomavirus DNA testing for cervical cancer screening. BMJ 2003;326:733.
16 Laverty CR, Farnsworth A, Thurloe JK, Grieves A, Bowditch R. Evaluation of the CytoRich slide preparation process. Anal Quant Histol 1997;19:239-45.

Accepted: 1 April 2007 